Vietnam Journal of Mechanics, VAST, Vol.38, No. 4 (2016), pp. 295-306

DOI:10.15625/0866-7136/7442

\title{
NUMERICAL SIMULATION OF SOLIDIFICATION AROUND A CIRCULAR CYLINDER WITH NATURAL CONVECTION
}

\author{
Vu Van Truong*, Truong Viet Anh, Hoang Thi Bich Ngoc \\ Hanoi University of Science and Technology, Vietnam \\ *E-mail: vuvantruong.pfae@gmail.com \\ Received November 21, 2015
}

\begin{abstract}
An analysis is carried out for solidification around a cold cylinder in a rectangular cavity using numerical simulations. The transient influences of solidification accompanied by natural convection are investigated in detail. The governing equations, in terms of one-fluid formulation, including the Navier-Stokes and energy equations for incompressible Newtonian fluids are written for the whole domain. The no-slip and Dirichlet-type isothermal temperature boundary conditions are both implemented using a linear interpolation technique (immersed boundary method). The solidification interface is represented by connected elements that move on the fixed background grid. Code validations are carried out through various problems. Finally, the temporal dependence of the solid area ratio of the solid phase to the cylinder upon various dimensionless parameters, such as Rayleigh number, Prandtl number, Stefan number, thermal property ratios as well as a parameter indicating the effect of superheat is studied.
\end{abstract}

Keywords: Solidification, cylinder, natural convection, front tracking, immersed boundary method.

\section{INTRODUCTION}

Solid-liquid phase change finds application in many systems and in nature including latent heat energy storage, metallurgy, and food and pharmaceutical processing. Solidification around cooled cylinders appears in thermal energy storage systems, freezing of soil and others. Therefore, understanding solidification heat transfer around cooled cylinders plays an important role in designing and operating such systems. Accordingly, there have been many works concerned with solidification around cylinders. Sasaguchi and co-workers [1] presented a numerical method for the solid-liquid phase change around a single cylinder and two horizontal cylinders in a rectangular cavity. They used a generalized coordinate system and their results were restricted to the freezing of water. A more complicated system in which there exist three or more cylinders has been investigated by Sugawara and Beer [2]. The authors used the PHOENICS code of a commercial software package. In another work [3], an enthalpy formulation-based

(C) 2016 Vietnam Academy of Science and Technology 
fixed-grid approach was employed to investigate the solidification around a number of staggered cylinders. However, in the above-mentioned papers, the results were restricted to water, and effects of some dimensionless parameters have not been considered.

In the present study, we present a numerical method for simulating the solidification around a cooled cylinder with natural convection. The method utilizes the front tracking technique [4] to represent the solid-liquid interface and an interpolation technique, i.e. an immersed boundary method, to deal with the no-slip and constant isothermal temperature boundary conditions. Effects of some dimensionless parameters are also investigated.

\section{MATHEMATICAL FORMULATION}

Fig. 1 shows the investigated problem, a solidification layer formed around a cooled circular cylinder in a rectangular cavity. The fluid and thermal properties of each phase are assumed constant, and the densities of the solid and liquid phases are identical (i.e. no volume change). In the liquid region, the flow assumed incompressible is driven by buoyancy-induced natural convection. We treat all phases as one fluid with variable properties such as density $\rho$, viscosity $\mu$, thermal conductivity $k$ and heat capacity $C_{p}$. In terms of the single-field representation, the momentum and thermal energy equations are

$$
\begin{gathered}
\frac{\partial}{\partial t}\left(\rho_{0} \mathbf{u}\right)+\nabla \cdot\left(\rho_{0} \mathbf{u u}\right)=-\nabla p+\nabla \cdot \mu\left(\nabla \mathbf{u}+\nabla \mathbf{u}^{T}\right)+\rho_{0} \mathbf{f}+\rho_{0} \mathbf{g}\left[1-\beta\left(T-T_{0}\right)\right] \\
\rho_{0} C_{p} \frac{\partial T}{\partial t}+\rho_{0} C_{p} \nabla \cdot(T \mathbf{u})=\nabla \cdot(k \nabla T)+\int_{f} \dot{q} \delta\left(\mathbf{x}-\mathbf{x}_{f}\right) d S+\rho_{0} C_{p} h \\
\nabla \cdot \mathbf{u}=0 .
\end{gathered}
$$

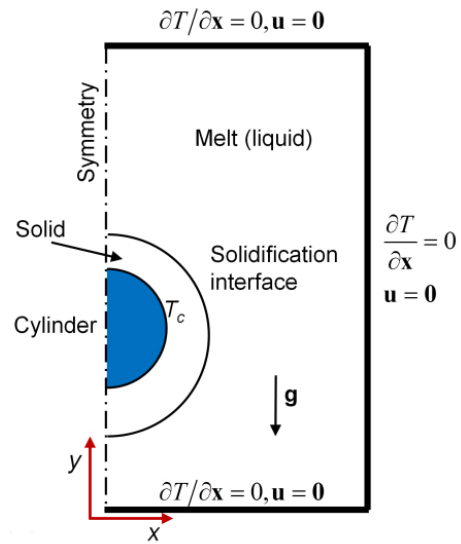

(a)

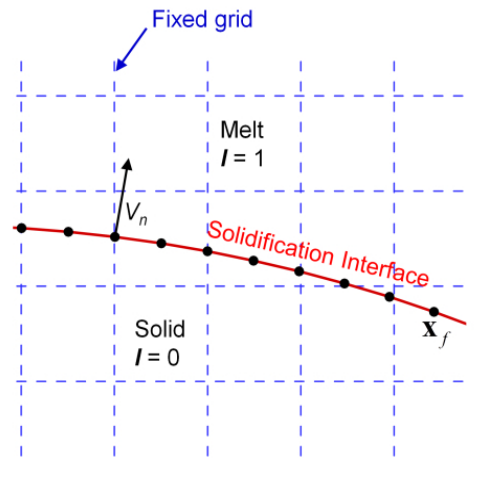

(b)

Fig. 1. Solidification around a cold cylinder in a rectangular cavity: (a) computational domain and (b) front-tracking representation for the solidification interface 
Here, $\mathbf{u}$ is the velocity vector, $p$ is the pressure, $\mathbf{g}$ is the gravitational acceleration. $T$ and the superscriptTdenote the temperature and the transpose. $D / D t$ is the material derivative. $\mathbf{f}$ is the momentum forcing used to impose no-slip condition on the solid-liquid interface [5]. The last term in Eq. (1) is the Boussinesq approximation for density changes due to thermal gradients [6]. $h$ is the energy forcing term used to impose a constant temperature on the cylinder boundary. $\rho_{0}$ is the density at the reference temperature $T_{0}$, e.g. fusion temperature. The Dirac delta function $\delta\left(\mathbf{x}-\mathbf{x}_{f}\right)$ is zero everywhere except for a unit impulse at the solidification interface $\mathbf{x}_{f}$. $\beta$ is the thermal expansion coefficient of the melt. $\dot{q}$ is the heat source at the solidification interface, given as

$$
\dot{q}=\left.k_{\mathrm{s}} \frac{\partial T}{\partial n}\right|_{\mathrm{s}}-\left.k_{1} \frac{\partial T}{\partial n}\right|_{1}=-\rho_{s} V_{n} L_{h},
$$

where the subscripts $s$ and $l$ represent solid and liquid, respectively. $V_{n}$ is the velocity normal to the solidification front (Fig. 1) and $L_{h}$ is the latent heat.

These above-mentioned equations are solved by the front-tracking method combined with interpolation techniques on a staggered grid with second order accuracy in time and space.

\section{NUMERICAL METHOD AND VALIDATION}

The numerical technique used in the present study is based on the front-tracking method [4] and an interpolation technique similar to the immersed boundary proposed in [5]. The momentum and mass conservation equations are discretized using an explicit predictor-corrector time-integration method and a second-order centered difference approximation for the spatial derivatives. The discretized equations are solved on a fixed, staggered grid using the MAC method [7]. The interface between the solid and liquid phases are represented by finite discrete points on a stationary grid, as shown in Fig. 1(b). These interface (or front) points propagate by

$$
\mathbf{x}_{f}^{n+1}=\mathbf{x}_{f}^{n}+\mathbf{n}_{f} V_{n} \Delta t
$$

where $V_{n}$ is simply given by

$$
V_{n}=-\dot{q}_{f} /\left(\rho_{s} L_{h}\right),
$$

and the heat source $\dot{q}$ is calculated using a normal probe technique $[8,9]$. Positions of the interface points are used to determine an indicator function $I$ which is zero in the solid phase and one the liquid phase

$$
\nabla I=\int_{f} \delta\left(\mathbf{x}-\mathbf{x}_{f}\right) \mathbf{n}_{f} d S,
$$

where $\mathbf{n}_{f}$ is the unit normal to the interface. Accordingly, $I=0.5$ corresponds to the interface. The indicator function $I$ is reconstructed from the new position of the interface points. The values of the material properties are then found: $\phi=\phi_{\mathrm{s}}(1-I)+I \phi_{1}$, where $\phi$ stands for $\rho, \mu, C_{p}$, or $k$. To impose no-slip condition on the solid-liquid interface as well as zero velocities within the solid phase, we use a method similar to the direct volume 
forcing method of Liao et al. [5]. We use a similar technique to determine the energy forcing inside, on and near the boundary to satisfy a constant temperature $T_{\mathcal{c}}$ on the cylinder surface. Detailed solution procedures, which based on the projection method, are as follows.

Suppose $n$ time steps have been completed, to calculate the solution at time level $n+1$ carry out the following steps:

1. Calculate the heat source, and update the solidification interface points (Eq. (5)).

2. Reconstruct the indicator function and update the material properties.

3. Calculate a provisional velocity field

$$
\mathbf{u}^{* *}=\mathbf{u}^{n}-\Delta t\left\{\nabla \cdot(\mathbf{u u})^{n}+\mu \nabla \cdot\left[\left(\nabla \mathbf{u}^{n}+\nabla\left(\mathbf{u}^{T}\right)^{n}\right)\right] / \rho_{0}+\mathbf{g}\left[1-\beta\left(T^{n}-T_{0}\right)\right]\right\} .
$$

4. Calculate a provisional temperature field

$$
T^{*}=\left[\rho_{0} C_{p}^{n} T^{n}+\Delta t\left(-\rho_{0} \nabla \cdot\left(C_{p}^{n} T^{n} \mathbf{u}^{n}\right)+\nabla \cdot\left(k^{n} \nabla T^{n}\right)+\int_{f} \dot{q} \delta\left(\mathbf{x}-\mathbf{x}_{f}\right) d S\right)\right] /\left(\rho_{0} C_{p}^{n+1}\right) .
$$

5. Establish locations of the momentum forcing points near the solidification interface and locations of the energy forcing points near the cylinder interface (see [5]for details).

6. Obtain the forcing term $\mathbf{f}$

$$
\mathbf{f}=\left(\mathbf{u}_{f}-\mathbf{u}^{* *}\right) / \Delta t
$$

with $\mathbf{u}_{f}$ is the velocity, at the forcing points, interpolated from $\mathbf{u}^{* *}$, or zero, at points inside the solid and cylinder [5].

7. Obtain the energy forcing term $h$

$$
h=\left(T_{f}-T^{*}\right) / \Delta t
$$

with $T_{f}$ is the temperature, at the energy forcing points near the cylinder interface, interpolated from $T^{*}$, or $T_{c}$, at the points inside the cylinder.

8. Update the temperature field at time $n+1$

$$
T^{n+1}=T^{*}+h \Delta t .
$$

9. Calculate an intermediate velocity field

$$
\mathbf{u}^{*}=\mathbf{u}^{* *}+\mathbf{f} \Delta t .
$$

10. Find the pressure field by solving the Poisson equation

$$
\left(\nabla \cdot \mathbf{u}^{n+1}-\nabla^{2} p\right) / \rho_{0}=-\left(\nabla \cdot \mathbf{u}^{*}\right) / \Delta t
$$

with $\nabla \cdot \mathbf{u}^{n+1}=0$.

11. Compute divergence-free time level $n+1$ fluid velocity

$$
\mathbf{u}^{n+1}=\mathbf{u}^{*}-\Delta t \nabla_{h} p / \rho_{0} .
$$


This solution procedure for time integration is first order, to produce a secondorder scheme, we use a technique described in Esmaeeli and Tryggvason [10] and in [11].

To validate the method, we first compare the computational results with exact solutions for a two-dimensional Stefan problem [12] in which a line heat source $Q$ causes a circular solid seed at the center to evolve in the direction of increasing the radius of the seed. This comparison is to validate the front-tracking method for solidification. Fig. 2 shows that the computational results agree well with the exact solutions at Stefan number $=0.1$, heat source $Q=40, C_{p s l}=0.5$ and $k_{s l}=2$. Detailed description of this comparison and others can be found in [4].

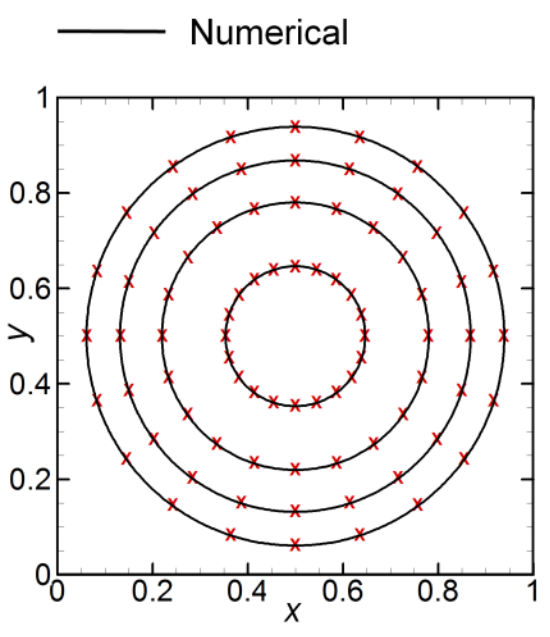

(a)
X Exact

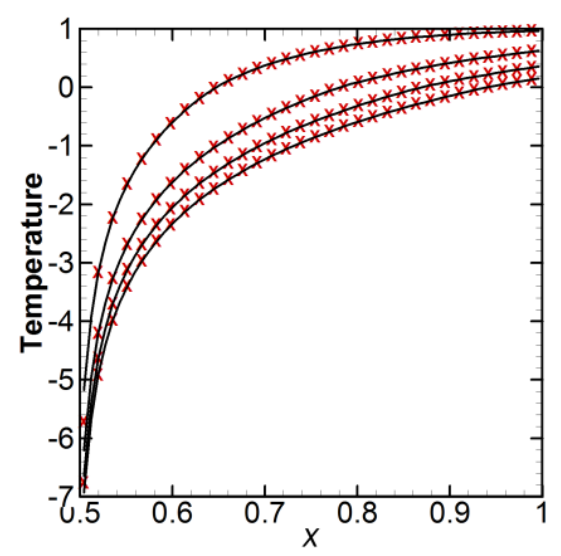

(b)

Fig. 2. Comparisons with exact solutions of Carslaw and Jaeger [12] for a 2D Stefan solidification problem: (a) evolution of the solidification front and (b) temperature along $y=0.5$. The data is plotted at time $t=0.03,0.11,0.19$ and 0.27

Next, to validate the technique of the momentum and energy forcing, we performed computations of natural convection in an annulus $[13,14]$. We use the same configuration and parameters as described in Wang and co-workers [14] (see Fig. 3). The inner cylinder has a radius $R_{i}=0.625$ and temperature $T_{h}$, while the outer cylinder has a radius $R_{0}=1.625$ and temperature $T_{c}$. The two cylinders are placed at the center of the $5 \times 5$ domain. The Prandtl number is $\operatorname{Pr}=0.7$ and the Rayleigh number is $R a=g \beta\left(T_{h}-T_{c}\right)\left(R_{0}-R_{i}\right)^{3} /(v \alpha)=5 \times 10^{4}$ where $v$ and $\alpha$ are the kinematic viscosity and the thermal diffusivity, respectively. The distribution of the temperature field in the annulus at the angle $\varphi=60^{\circ}$ is shown in Fig. 3(b). Good agreement between our calculation and the result from Kuehn and Goldstein [13], as well as the result of Wang et al. [14] (see Fig. 3(b)) supports the accuracy of the present method. 


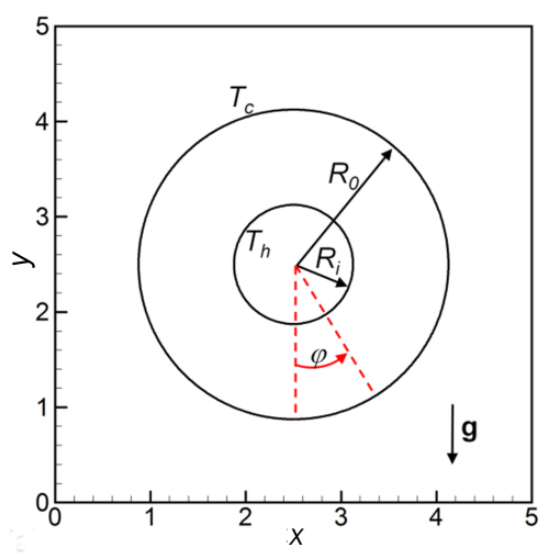

(a)

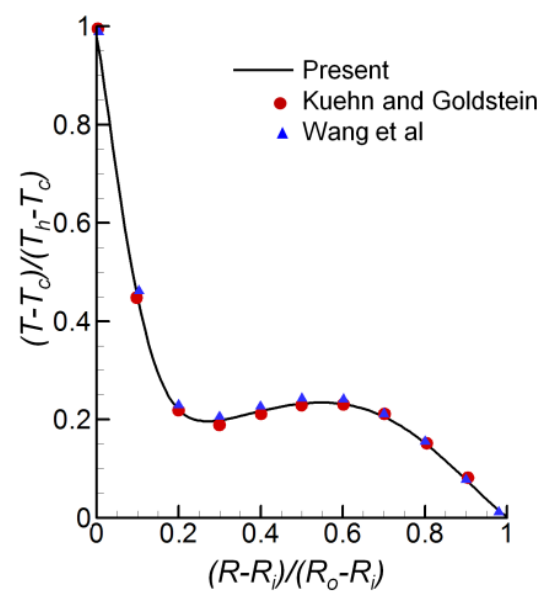

(b)

Fig. 3. Natural convection in an annulus: (a) domain for computation and (b) temperature variation in the annulus at $\varphi=60^{\circ}$ in comparison with data reported in Kuehn and

Goldstein [13] and in Wang et al. [14]

\section{RESULTS AND DISCUSSION}

We turn to our main problem, i.e. solidification around a circular cylinder (Fig. 1). We choose the diameter $d$ of the cylinder as a scaling length, and $\tau_{c}=\rho_{1} C_{\mathrm{pl}} d^{2} / k_{1}$ as the characteristic time scale. The characteristic velocity scale is thus taken to be $U_{\mathrm{c}}=$ $d / \tau_{\mathrm{c}}$. With these above choices, it is possible to show that the dynamics of the problem is governed by the Prandtl number $P r$, the Rayleigh number $R a$, the dimensionless initial temperature in the liquid $\theta_{0}$, and the ratios of the thermal properties of the solid to liquid

$$
S t=\frac{C_{p l}\left(T_{m}-T_{c}\right)}{L_{h}}, \operatorname{Pr}=\frac{C_{p l} \mu_{l}}{k_{l}}, R a=\frac{g \beta\left(T_{i}-T_{m}\right) d^{3}}{v_{l} \alpha_{l}}, \theta_{0}=\frac{T_{i}-T_{c}}{T_{m}-T_{c}}, C_{p s l}=\frac{C_{p s}}{C_{p l}}, k_{s l}=\frac{k_{s}}{k_{l}} .
$$

The temperature is non-dimensionalized as $\theta=\left(T-T_{c}\right) /\left(T_{m}-T_{c}\right)$. The dimensionless time is $\tau=t / \tau_{c}$. The center of the cylinder is at $\mathbf{x} / d=(0,3)$ in a domain of $2 \times 6$. For parametric investigations of phase change problems, the values of these parameters are varied in various ranges. For example, for liquid metals or semiconductors $P r$ is varied in the range of 0.01 to $0.1, R a$ is varied in the range of $10^{3}$ to $10^{5}$, St is varied in the range of 0.01 to 1.0 , and $\theta_{0}$ is varied in the range of 0.1 to 2.0 [15-17]. Accordingly, in the present study, the typical values of the parameters are chosen as $S t=0.1, \operatorname{Pr}=0.1, R a=1 \times 10^{5}, C_{p s l}=1.0, k_{s l}=1.0$, and $\theta_{0}=2$. These values correspond to a liquid metal $[15,16]$ or a semiconductor material [17].

We first perform grid refinement study for our solidification problem (Fig. 1) to choose the best grid. To do so, we perform simulations with the typical values of the parameters, i.e. $S t=0.1, \operatorname{Pr}=0.1, R a=1 \times 10^{5}, C_{p s l}=1.0, k_{s l}=1.0$, and $\theta_{0}=2$, 
for three grid resolutions $64 \times 192,128 \times 384$ and $256 \times 768$ as shown in Fig. 4. Fig. 4(a) shows the variation with respect to time of the ratio $A s / A c$ of the solid phase area to the cylinder area. Comparison of the front locations obtained with two finer grids is shown in Fig. 4(b). As shown in this figure, the grid $64 \times 192$ yields the results different from those obtained by the two finer grids. Contrarily, the results obtained from $128 \times 384$ and from $256 \times 768$ are almost identical. Accordingly, we use $128 \times 384$ for all computations presented below.

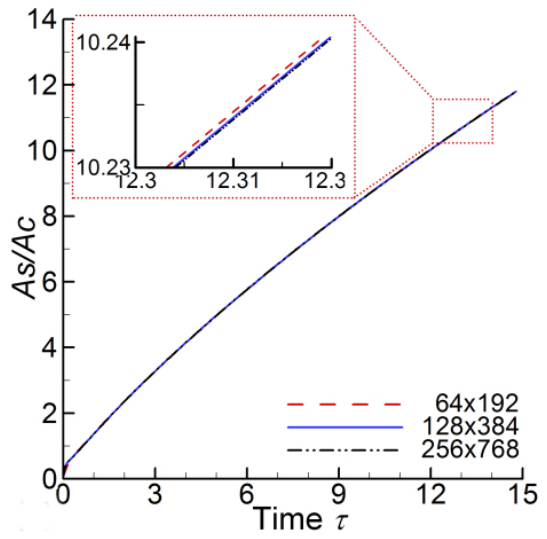

(a)

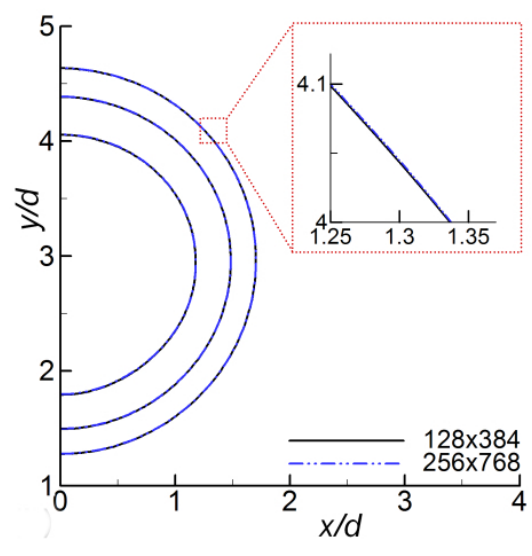

(b)

Fig. 4. Grid refinement study with $S t=0.1, \operatorname{Pr}=0.1, R a=1 \times 10^{5}, C_{p s l}=1.0, k_{s l}=1.0$, and $\theta_{0}=2$ : (a) temporal variation of the area ratio of the solid phase to the cylinder (As/Ac); and (b) location of the solidification front at time $\tau=4.2,8.4$ and 12.6

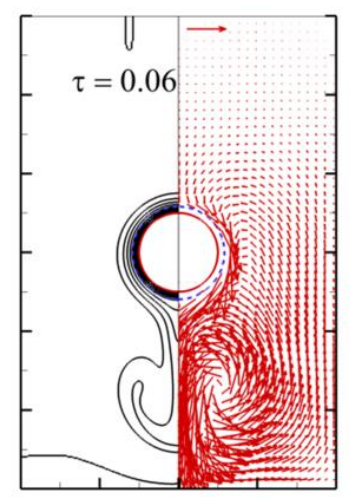

(a)

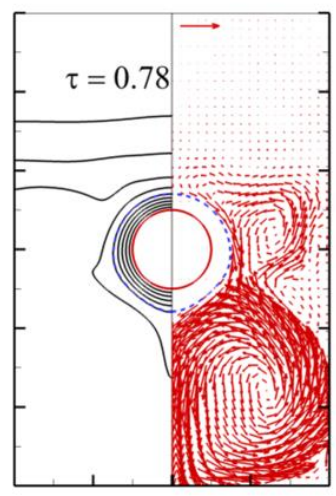

(b)

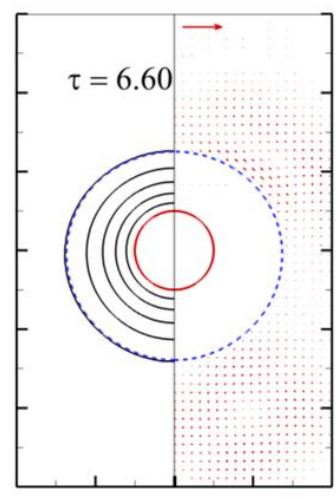

(c)

Fig. 5. Solidification around a cylinder with $S t=0.1, P r=0.1, R a=1 \times 10^{5}, C_{p s l}=1.0, k_{s l}=1.0$, and $\theta_{0}=2$. In each frame, the left shows the isotherms (plotted every $\Delta \theta=0.4$ ) and the right shows the velocity field normalized by $U_{c}$ (the reference vector has a magnitude of 50).

The dash line is the solidification front 
Fig. 5 shows the temporal evolution of the solidification front with temperature and velocity fields. At early times of the solidification process, downward flow arises along the solid-liquid interface (the dash line in Fig. 5), at which the temperature is $\theta_{m}=1.0$, because the density of the liquid increases with a decrease in the temperature. As shown in Fig. 5(a) and 5(b), the downward flow is strong with the cooled liquid accumulating at the bottom part of the cavity. As a result, a thermally stratified region is gradually formed. As time progresses, the temperature of the liquid phase decreases to near the fusion temperature $\theta_{m}$. Consequently, the downward flow is suppressed. At $\tau=6.6$ (Fig. 5(c)), the liquid temperature is uniform in the entire cavity, and almost no flows are evident at this time, and the solidification process is merely controlled by conduction. As shown in Fig. 5(a) and 5(b) that the solidification growth rate is slightly faster around the lower part of the cylinder due to the thermal stratification.

Next we consider the effects of some parameters on the solidification process.

\subsection{Effects of the initial temperature}

Fig. 6(a) compares the velocity field in the lower part of the cavity for two cases: $\theta_{0}=1.25$ (left) and 2.25 (right) at an early time of the solidification process, i.e. $\tau=1.68$. It is evident that the solidification rate for $\theta_{0}=2.25$ is smaller than that for $\theta_{0}=1.25$. This is understandable since the warm liquid flows more strongly towards the solidification interface for $\theta_{0}=2.25$ than it does for $\theta_{0}=1.25$ (Fig. 6(a)). As a result, smaller initial liquid temperatures lead to larger solid area ratios $A s / A c$ as shown in Fig. 6(b) .

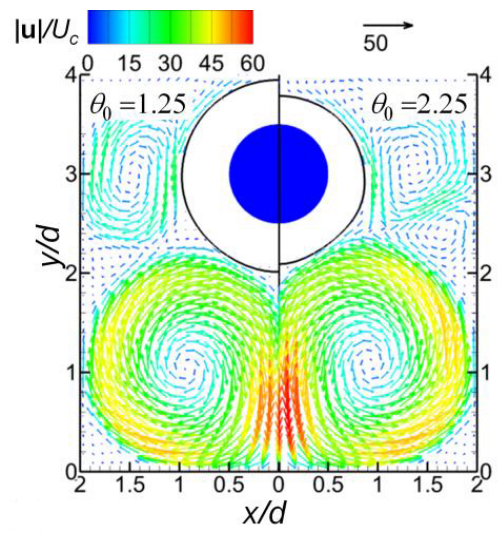

(a)

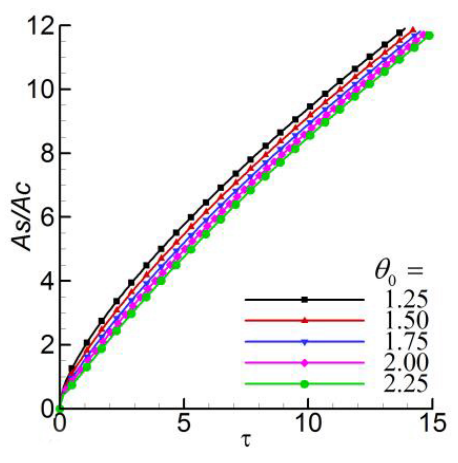

(b)

Fig. 6. Effects of the initial temperature on the solidification: (a) velocity field at $\tau=1.68$ for $\theta_{0}=1.25$ (left) and $\theta_{0}=2.25$ (right); (b) evolution of the solid area ratio.

$$
S t=0.1, \operatorname{Pr}=0.1, R a=1 \times 10^{5}, C_{p s l}=1.0 \text {, and } k_{s l}=1.0
$$

\subsection{Effects of $R a$ and $P r$}

Fig. 7(a) shows the effect of $R a$ on the solidification process. At early times, as $R a$ increases the warm fluid flows more strongly towards the solidification interface, resulting in a decrease in the solidification rate (see the lower set in Fig. 7(a)). However, strong 
circulation corresponding to high values of $R a$ causes liquid temperature to fast reach the fusion temperature of the solidification interface. Accordingly, as time progresses the solidification rate becomes larger for higher $\mathrm{Ra}$ (the upper set in Fig. 7(a)). Consequently, the solid area ratio $(A s / A c)$ is larger for higher $R a$ as shown in Fig. 7(a).

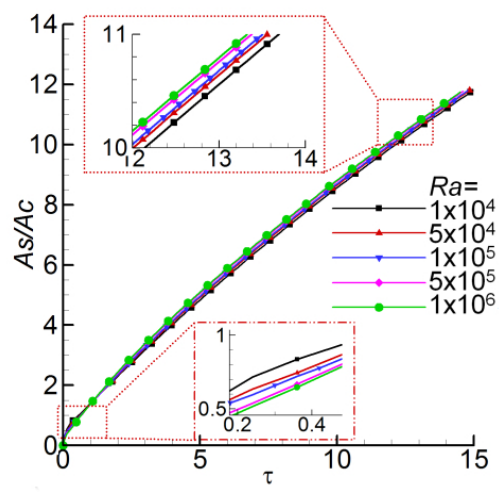

(a)

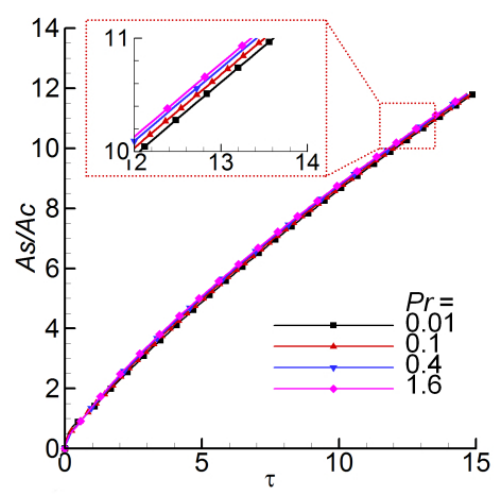

(b)

Fig. 7. Effects of (a) the Rayleigh number with $\operatorname{Pr}=0.1$, and (b) the Prandtl number with $R a=1 \times 10^{5}$. Other parameters: $S t=0.1, C_{p s l}=1.0, k_{s l}=1.0$, and $\theta_{0}=2$

The influence of the Prandtl number $\operatorname{Pr}$ on the area ratio is shown in Fig. 7(b). As $\mathrm{Pr}$ increases the solid area ratio increases. This is understandable since increasing $\mathrm{Pr}$ corresponds to increasing the viscous effect. Accordingly, the warm liquid flows more strongly towards the solidification interface at smaller $\mathrm{Pr}$, resulting in a decrease in the solidification rate (Fig. 7(b)).

\subsection{Effects of $S t$ and $k_{s l}$}

The effect of St on the solidification process is shown in Fig. 8(a). According to Eq. (6), increasing St corresponding to decreasing the latent heat released (see Eq. (16)) results in an increase in the growth rate of the solidification front, and thus increases the solid area ratio.

We expect that the solidification process is considerably affected by the solid-toliquid thermal conductivity ratio $k_{s l}$. Fig. 8(b) confirms our expectation. According to Eq. (4), a higher solid-to-liquid thermal conductivity ratio produces higher heat flux at the solidification interface. Thereby, increasing this ratio results in an increase in the solidification rate. As a result, the solid area ratio is larger at higher $k_{s l}$, as clearly shown in Fig. 8(b).

\subsection{Effects of $C_{p s l}$}

A comparison of velocity vectors in the lower part of the cavity (at $\tau=1.8$ ) for $C_{p s l}=0.5$ (left) with those for $C_{p s l}=1.5$ (right) is shown in Fig. 9(a). We see two circulations on the left $\left(C_{p s l}=0.5\right)$ while stronger ones appear on the right $\left(C_{p s l}=1.5\right)$. In other words, the warm fluid flows more strongly towards the solidification interface for 


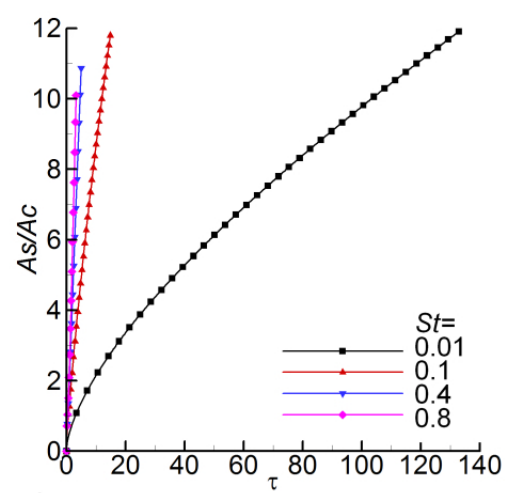

(a)

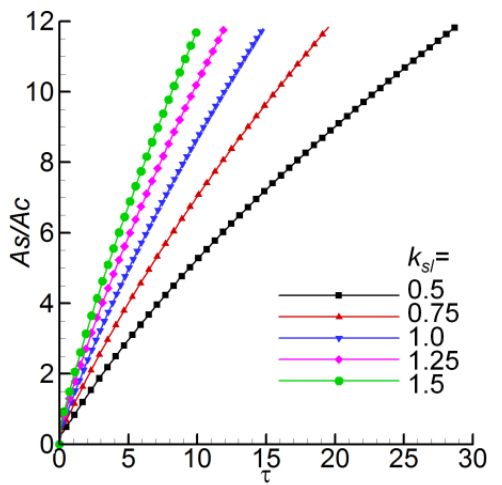

(b)

Fig. 8. Effects of (a) the Stefan number with $k_{s l}=1.0$, and (b) the solid-to-liquid thermal conductivity ratio $k_{s l}$ with $S t=0.1$. Other parameters:

$$
R a=10^{5}, \operatorname{Pr}=0.1, C_{p s l}=1.0 \text { and } \theta_{0}=2
$$

higher $C_{p s l}$, resulting in a decrease in the solidification rate. Accordingly, the solid-liquid interface proceeds faster as $C_{p s l}$ decreases. The solid area ratio is thus smaller at higher $C_{p s l}$, as clearly shown in Fig. $9(\mathrm{~b})$.

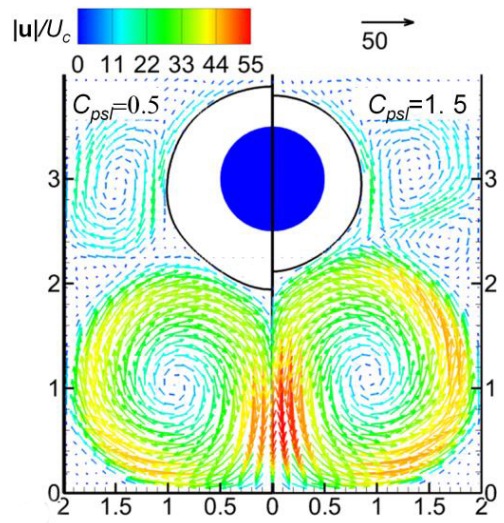

(a)

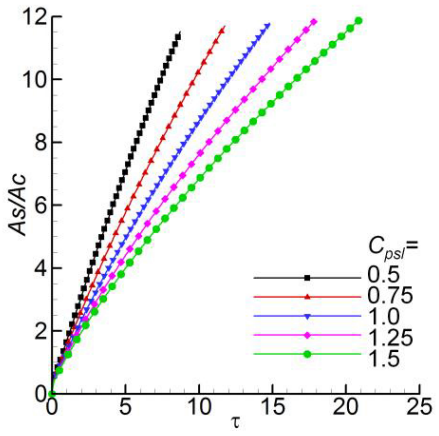

(b)

Fig. 9. Effects of the solid-to-liquid heat capacity ratio $C_{p s l}$ with $S t=0.1, R a=10^{5}, P r=0.1$, $k_{s l}=1.0$, and $\theta_{0}=2$ : (a) velocity field normalized by $U_{c}$ at $\tau=1.8$ presented for $C_{p s l}=0.5$ (left) and for $C_{p s l}=1.5$ (right); and (b) temporal variation of the solid area ratio

\section{CONCLUTION}

An investigation has been carried out for the solidification, under the influence of natural convection, around a cooled cylinder in a rectangular enclosure. The numerical 
method including the front-tracking technique representing for the solidification interface and an immersed boundary technique for the no-slip and Dirichlet-type isothermal temperature boundary conditions has been used. The Navier-Stokes and enery equations have been solved on the whole domain with the fixed and rectangular grid. The method has been validated by comparisons with the exact solutions for a 2D Stefan solidification problem, and with the natural convection solutions in an annulus reported in $[13,14]$.

For the problem of solidification around a cylinder, the influences of various dimensionless parameters on the solidification process have been investigated. Numerical results show that increasing $R a, P r, S t$ or $k_{s l}$ increases the solidification rate. Contrarily, the solidification interface is found to proceed faster as the initial liquid temperature $\theta_{0}$ or $C_{p s l}$ decreases.

\section{ACKNOWLEDGMENT}

This research is funded by Vietnam National Foundation for Science and Technology Development (NAFOSTED) under grant number 107.03-2014.21. We are grateful to Prof. John C. Wells at Ritsumeikan University (Japan) for facilitating our computing resources.

\section{REFERENCES}

[1] K. Sasaguchi, K. Kusano, and R. Viskanta. A numerical analysis of solid-liquid phase change heat transfer around a single and two horizontal, vertically spaced cylinders in a rectangular cavity. International Journal of Heat and Mass Transfer, 40, (6), (1997), pp. 1343-1354. doi:10.1016/s0017-9310(96)00210-4.

[2] M. Sugawara and H. Beer. Numerical analysis for freezing/melting around vertically arranged four cylinders. Heat and Mass Transfer, 45, (9), (2009), pp. 1223-1231. doi:10.1007/s00231-009-0492-y.

[3] Y.-C. Shih and H. Chou. Numerical study of solidification around staggered cylinders in a fixed space. Numerical Heat Transfer, Part A: Applications, 48, (3), (2005), pp. 239-260. doi:10.1080/10407780590945579.

[4] T. V. Vu, G. Tryggvason, S. Homma, J. C. Wells, and H. Takakura. A front-tracking method for three-phase computations of solidification with volume change. Journal of Chemical Engineering of Japan, 46, (11), (2013), pp. 726-731. doi:10.1252/jcej.13we169.

[5] C.-C. Liao, Y.-W. Chang, C.-A. Lin, and J. McDonough. Simulating flows with moving rigid boundary using immersed-boundary method. Computers \& Fluids, 39, (1), (2010), pp. 152 167. doi:10.1016/j.compfluid.2009.07.011.

[6] H. Gan, J. Chang, J. J. Feng, and H. H. Hu. Direct numerical simulation of the sedimentation of solid particles with thermal convection. Journal of Fluid Mechanics, 481, (2003), pp. 385-411. doi:10.1017/s0022112003003938.

[7] F. H. Harlow, J. E. Welch, et al. Numerical calculation of time-dependent viscous incompressible flow of fluid with free surface. Physics of fluids, 8, (12), (1965), pp. 2182-2189. doi:10.1063/1.1761178.

[8] N. Al-Rawahi and G. Tryggvason. Numerical simulation of dendritic solidification with convection: two-dimensional geometry. Journal of Computational Physics, 180, (2), (2002), pp. 471496. doi:10.1006/jcph.2002.7092. 
[9] A. Esmaeeli and G. Tryggvason. Direct numerical simulations of bubbly flows. part 1. low reynolds number arrays. Journal of Fluid Mechanics, 377, (1998), pp. 313-345. doi:10.1017/s0022112098003176.

[10] A. Esmaeeli and G. Tryggvason. Computations of film boiling. part i: numerical method. International Journal of Heat and Mass Transfer, 47, (25), (2004), pp. 5451-5461. doi:10.1016/j.ijheatmasstransfer.2004.07.027.

[11] G. Tryggvason, R. Scardovelli, and S. Zaleski. Direct numerical simulations of gas-liquid multiphase flows. Cambridge University Press, (2011). doi:10.1017/cbo9780511975264.

[12] H. S. Carslaw and J. C. Jaeger. Conduction of heat in solids. Oxford University Press, USA, (1986).

[13] T. H. Kuehn and R. J. Goldstein. An experimental and theoretical study of natural convection in the annulus between horizontal concentric cylinders. Journal of Fluid Mechanics, 74, (04), (1976), pp. 695-719. doi:10.1017/s0022112076002012.

[14] Z. Wang, J. Fan, K. Luo, and K. Cen. Immersed boundary method for the simulation of flows with heat transfer. International Journal of Heat and Mass Transfer, 52, (19), (2009), pp. 45104518. doi:10.1016/j.ijheatmasstransfer.2009.03.048.

[15] N. Ramachandran, J. Gupta, and Y. Jaluria. Thermal and fluid flow effects during solidification in a rectangular enclosure. International Journal of Heat and Mass Transfer, 25, (2), (1982), pp. 187-194. doi:10.1016/0017-9310(82)90004-7.

[16] D. Johnson and R. Narayanan. A note on the stability of a solidifying material in an undercooled melt in the presence of convection. In Interactive Dynamics of Convection and Solidification, pp. 25-32. Springer, (2001). doi:10.1007/978-94-015-9807-1_4.

[17] B. T. Murray, S. R. Coriell, G. B. McFadden, A. A. Wheeler, and B. V. Saunders. Gravitational modulation of thermosolutal convection during directional solidification. Journal of Crystal Growth, 129, (1), (1993), pp. 70-80. doi:10.1016/0022-0248(93)90435-y. 